\title{
An Auxiliary Classification Diagnosis Software System of Liver Medical Data Based on Various Artificial Neural Networks
}

\author{
Yong Qi ${ }^{\text {a) }}$, Wenyue Gou ${ }^{\text {b) }}$, Jie $\mathrm{Li}^{\text {c) }}$, Qiaosheng Zhu ${ }^{\text {d) }}$ and Runze Pei ${ }^{\text {e) }}$ \\ Shaanxi University of Science and Technology, Shaanxi, 710021, China. \\ a) qiyong@sust.edu.cn \\ b) Corresponding author: Jessicafield201@gmail.com \\ c)a18066528696@gmail.com \\ d)1102022941@qq.com \\ e) 53704723@qq.com
}

\begin{abstract}
This paper proposed to develop an auxiliary software system for recognizing the medical data of hepatic pathological changes based on the deep learning of medical data identification technology.Built under the idea of personal demands and self-service platform, the system is supported by artificial intelligence, data mining, network and other techniques. Focusing on the core needs of clinical diagnosis and medical data identification technology, we constructed several major modules for liver diagnosis and treatment information acquisition, knowledge dissemination and so on. The method effectively solved the problem of non-standardization and non-precision shortcomings in the process of recognizing the medical data of hepatic pathological changes. More importantly, it can better cope with current problems such as workload, low recognition accuracy lack of strong expertise and requirements of manpower and resources.
\end{abstract}

Key words: liver complaint; machine learning; deep learning; medical support platform.

\section{INTRODUCTION}

In modern medical technology field, medical imaging and medical data sets are indispensable for the diagnosis and recognition of liver diseases. The two methods are widely used in clinical diagnosis and have important clinical application in various aspects, such as surgical planning, surgical navigation, the tracking of pathological changes and the evaluation of therapeutic effects.

However, due to the large amount of data sets and the large number of irregular and incomplete data, it is quite difficult to determine whether an individual in multiple groups is affected by lesions. With the development of artificial intelligence, the method of using machine learning assistant to diagnose is gradually preferred by the public. Han et al. proposed an automatic classification algorithm of liver fibrosis based on machine learning. Through categorizing the images into normal case(f0-f1) and abnormal (f2-f4) case, cirrhosis case(F4) and non-cirrhosis case(f0-f3), they obtained the accuracy of $89.10 \%$ and $91.64 \%$ respectively based on the tenfold cross-validation. Lee promoted an automatic liver tumor segmentation method based on convolutional neural networks (CNN). This method enables the network to study the features automatically and allows more separability. Thus, it is more able to depict the rich inner information of data and facilitate tumor segmentation towards higher accuracy and robustness.

This paper introduced a hepatic pathological changes medical data recognition and diagnosis auxiliary system, which can properly solve the difficulties of over-concentrated medical data, heavy workload and large error. The software system can be applied to most current medical operating system, so that the relevant workers can collect, analyze and process relevant data and gain the final diagnosis. 


\section{EXPERIMENTS}

The medical aided diagnosis platform developed by our team is an artificial intelligence-based auxiliary diagnosis platform. It is divided into three sections: network training, user diagnosis, and network self-improvement.

(1) Network training:

This section extracted the dataset of hepatopathy diagnosed by experts from the database. Then, the optimal algorithm for parameter training is applied to the constructed machine learning network and neural network.

The machine learning network adopted SVM (Support Vector Machine) model and KNN (k-nearest neighbor) algorithm respectively. The SVM maps the sample space to a high-dimensional or even infinite dimensional feature space through a nonlinear mapping, so that the problem of nonlinear separability in the original sample space is transformed into a linearly separable problem in the feature space. The idea of KNN method: if a sample has the $\mathrm{k}$ most similar (i.e. the nearest neighbor in the feature space) samples in a feature space belonging to a certain category, the sample also belongs to this category.

The neural network adopted FNN (feedforward neural network). Each neuron is connected to the neurons only in the previous layer. It operates by receiving the output of the previous level, and outputting to the next level. There is no feedback between layers, which can be represented by a directed acyclic graph. It is currently the most widely used and the one of the fastest growing artificial neural network.

After a certain training for the three networks, the network was used to predict whether the patients were diseased according to the test data. The training was completed when the recognition accuracy reached a certain degree. The results showed that the neural network was the most accurate, thus the neural network was applied to the system.

(2) User diagnosis:

This section had the trained network model used in the real diagnosis. Firstly, the user typed-in the related information and examination results via the human-computer interaction interface. Then the system would preprocess the entered information. It diagnosed by testing the processed data through a trained neural network. If there is no disease, the system terminates and outputs a corresponding report description based on the structure; if the diagnosis is a disease, a report is generated based on the result and a further examination by the doctor is required. Finally, the user should be diagnosed on the basis of doctor's advice.

(3) Network self-improvement:

This section is to introduce the further diagnostic results of the previous section into the database of liver disease patients by doctors, mark them with related labels, and retrain the previously trained neural network regularly (when the newly added data reaches a certain number). The self-improvement of the network itself will further improve the accuracy of the diagnostic results, making the verification platform more reliable and prolonging the system life.

We did an external delivery test of the medical auxiliary diagnosis platform. The flow chart is as Fig.1. 


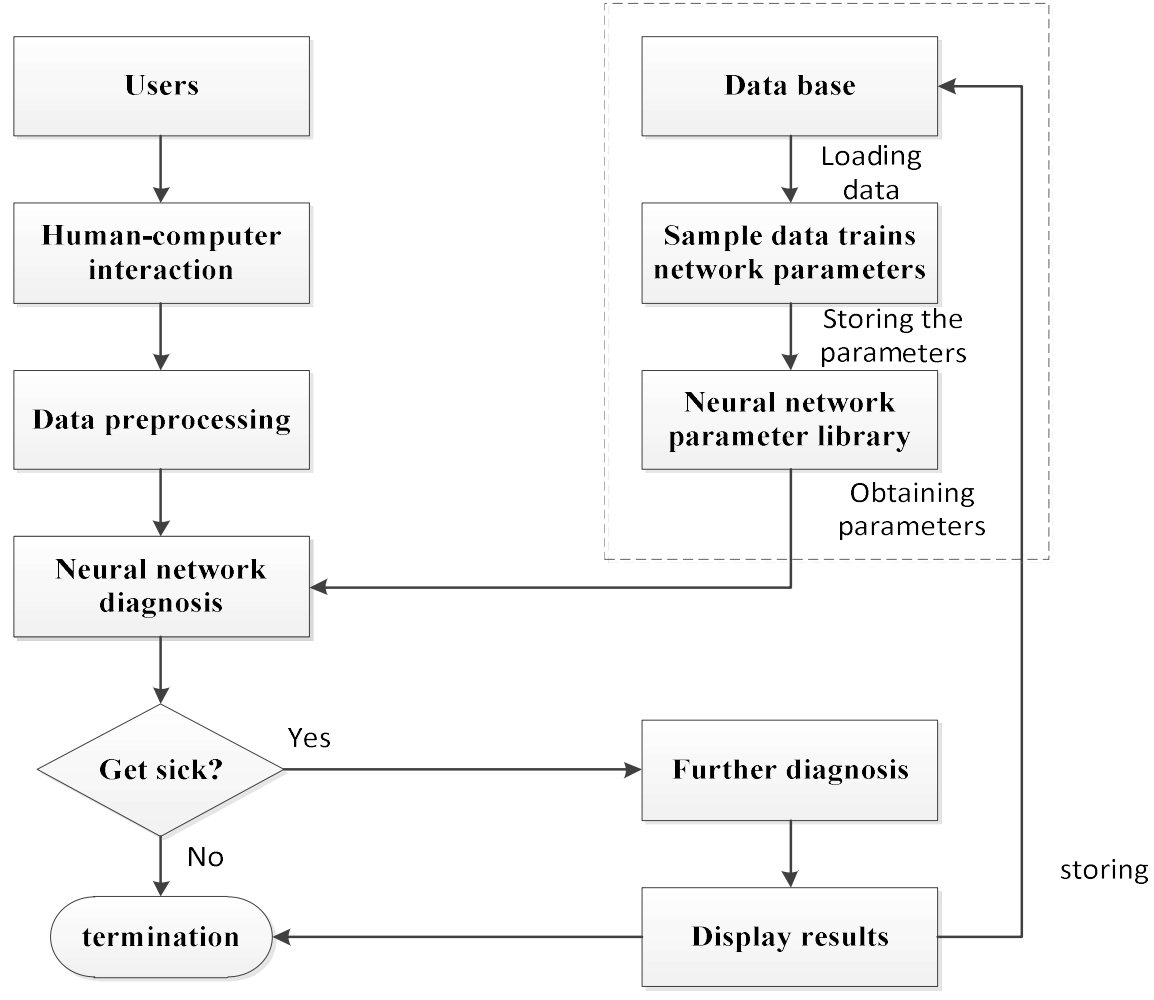

FIGURE 1. Flow chart

\section{ANALYSIS}

After full testing, the medical auxiliary diagnostic platform has equipped with a certain amount of data, and the results of the system network have almost reached the optimal situation. The system can be further improved and put into the market for use.

\section{CONCLUSION}

The software system introduced in this article can effectively extract features of liver medical data and make more accurate classification and diagnosis. The proposed model based on SVM, FNN and KNN combined with liver medical diagnosis availably improved the efficiency and accuracy of physicians in the diagnosis of liver medical data and provided an innovative method to gradually improve the level of intelligent diagnosis way.

\section{ACKNOWLEDGEMENTS}

We are grateful to the following funds:

Student's Platform for Innovation and Entrepreneurship Training Program

(1303)

Education Department of Shaanxi

Province

(15JK1086)

Natural Science Foundation of Shaanxi

Province

(2015GY009)

Shaanxi University of Science and

Technology Dr. Foundation 
(BJ14-07)

\section{REFERENCES}

1. Han. Ultrasound images of liver fibrosis in machine learning method based on automatic classification of [D]. of Shenzhen University, 2016.

2. Li Wen. Study on the segmentation of liver tumor in CT image based on deep convolution neural network [D]. University of Chinese Academy of Sciences, 2016.

3. Experimental data: https://www.kaggle.com/jeevannagaraj/indian-liver-patient-dataset/data

4. Schuwei, schuke, PAM, et al, et al. The application of machine learning in early diagnosis and prognosis of cancer [J]. Journal of medical informatics, 2016, 37 (11): 10-14.

5. Huang Shujing, Lou gemtek. Technology application of $[\mathrm{J}]$. staining analysis and clinical diagnosis of hepatocellular carcinoma, 2016, 23 (4): 466-469.

6. Shi, Haobin*, Lin, Zhiqiang, Hwang, Kao-Shing, Yang, Shike, Chen, Jialin, An Adaptive Strategy Selection Method with Reinforcement Learning for Robotic Soccer Games, IEEE Access, Volume 6, 2018(SCI, IF:3.244, area 2, DOI:10.1109/ACCESS.2018.2808266)

7. Haobin Shi, Xuesi Li, Kao-Shing Hwang, Wei Pan, Genjiu Xu, Decoupled Visual Servoing with Fuzzy QLearning IEEE Transactions on Industrial Informatics Volume 14; Issue 1, PP:241-252, 2018(SCI, IF:6.764,1 Area, TOP Periodical)

8. Wang Ranran. Research on machine learning for disease intelligent diagnosis [D]. East China Jiaotong University, 2015. 